Gut, 1984, 25, 854-862

\title{
Multiple and recurrent inflammatory fibroid polyps in three generations of a Devon family: a new syndrome
}

\author{
P P ANTHONY, D S MORRIS, AND K D J VOWLES \\ From the Departments of Pathology and Surgery, Postgraduate Medical School, University of Exeter, and \\ Royal Devon and Exeter Hospital, Exeter, Devon, and Green Lane Hospital, Auckland, New Zealand
}

SUMMARY The recurrence of multiple inflammatory fibroid polyps is reported in three generations of a Devon family. Only one female in each has been affected in a direct line of descent. The grandmother has had nine polyps resected over 11 years, the mother seven over 18 years, and the daughter six over six years. None of the patients or their relatives are known to have any allergies, dietary fads, or gastrointestinal infections. Chromosome studies have been normal in two patients. No cancer risk has been identified. Conventional histology, electron microscopy, and immunohistology suggest that the lesion is a self-limiting proliferation of histiocytes; the initiating event or stimulus remains unknown. A genetic factor is likely to operate in this unique family which is probably polygenic and multifactorial in nature.

Polyposis of the gastrointestinal tract is a complex subject that is now gradually becoming clarified. ${ }^{1}$ The term is usully meant to exclude benign soft tissue tumours such as lipoma of the ileocaecal region or rectum, leiomyoma of stomach or intestine and gastrointestinal involvement in the autosomal dominant conditions of von Recklinghausen's disease and the blue rubber bleb naevus syndrome. ${ }^{2}$ Leaving these aside, one is left with a heterogenous group of lesions characterised by their multiplicity, epithelial origin, and a risk of malignancy. Well known examples include familial colonic polyposis, Gardner's syndrome, juvenile polyposis, PeutzJeghers' syndrome, and Turcot's syndrome. All these are, or can be, familial, the mode of inheritance being autosomal dominant with the possible exception of Turcot's syndrome. Their nature, manifestations, and genetics have been extensively reviewed in recent years. ${ }^{2-4}$

The lesion known as inflammatory fibroid polyp stands alone in this field. It was first described by Vanek in $1949^{5}$ as a 'gastric submucosal granuloma with eosinophilic infiltration' but many names have been applied to it since: inflammatory pseudotumour, eosinophilic granuloma, fibroma, haemangiopericytoma, schwannoma, that reflect the varying views about its nature. The term inflam-

Address for correspondence: Dr P P Anthony, University of Exeter Postgraduate Medical School, Banack Road, Exeter EX2 5DW.

Received for publication 11 November 1983 matory fibroid polyp' was coined by Helwig and Ranier ${ }^{6}{ }^{7}$ and has become the one most widely used. Johnstone and Morson ${ }^{8}$ described 13 cases and reviewed the literature on a further 76 ; Nambu and colleagues ${ }^{9}$ reported 40 cases of which 21 were considered 'early'. Apart from these two series, the literature consists of single case reports or reports of two or three cases only: the lesion is rare.

Characteristically, an inflammatory fibroid polyp is a solitary tumour like mass in the stomach and is rarely found elsewhere. It consists of loosely organised, vascular and fibrous tissue with a variable number of eosinophils. Differentiation from diffuse forms of eosinophilic infiltration of the gastrointestinal tract is important. ${ }^{10}$ Multiple polyps have been recorded only once and were one each in the duodenum and jejunum. ${ }^{11}$ Recurrence or a familial occurrence have not been encountered up to now.

We wish to present our findings based on a large Devon kindred in whom multiple and recurrent inflammatory fibroid polyps developed in three successive generations.

\section{Methods}

The clinical findings in each of the three patients are reported below. All the living relatives were interviewed regarding diet, allergies, and a complete medical history was obtained; the family tree is presented (Fig. 1). Histological material from all 


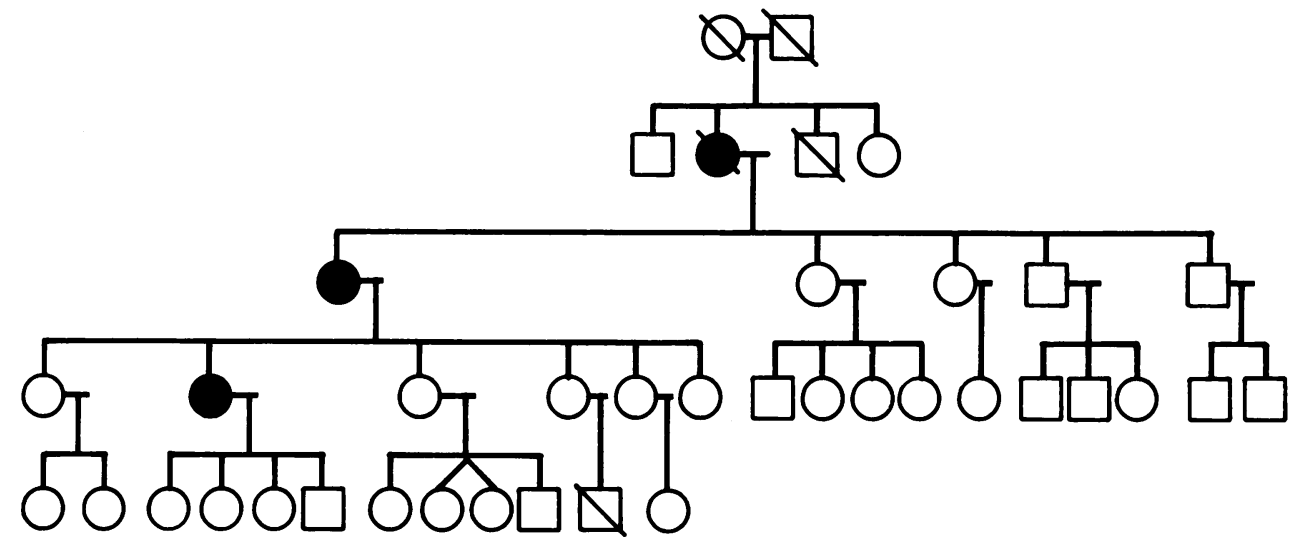

Fig. 1 Family tree: males are represented by open squares and females by open circles. The three patients, all females, are indicated by solid circles. The diagonal line identifies those who are now dead.

surgical specimens has been collected, sectioned, and stained with haematoxylin-eosin, reticulin, martius-scarlet-blue, phosphotungstic acidhaematoxylin, thionin, and chloracetate esterase. Two polyps from case 2 were examined by electron microscopy. Two polyps and intestinal mucosa from each of the three cases were further examined by the PAP modification of the immunoperoxidase method $^{12}$ for immunoglobulins $\mathrm{G}, \mathrm{A}, \mathrm{M}$, and $\mathrm{E}$, kappa and lambda light chains, lysozyme and $\alpha_{1}$-antitrypsin using commercially available antisera. Apart from minor variations, the pathological findings were identical in all the polyps and they are described together.

\section{Case reports}

The salient features are summarised in the Table.

CASE 1

Mrs MS first presented in 1967 when she was 59 years of age. Her symptoms were recurrent colicky abdominal pain and vomiting. At laparotomy an ileo-ileal intussusception was found due to a $6.0 \mathrm{~cm}$ submucosal polyp $25.0 \mathrm{~cm}$ from the duodenojejunal junction. A small bowel resection was carried out. She was admitted to hospital again in 1969 with a six months' history of abdominal pain associated with vomiting. A barium meal suggested polyps in the gastric antrum. A $1.0 \mathrm{~cm}$ submucosal polyp was found and resected, together with a $1.5 \mathrm{~cm}$ polyp in the terminal ileum.

In 1974 she experienced episodes of epigastric pain relieved by vomiting. Barium meal and gastroscopy showed two separate gastric antral polyps, each $0.5 \mathrm{~cm}$. These were resected. Again, a $0.5 \mathrm{~cm}$ polyp was found in the mid-ileum and this, too, was resected.

She presented as an abdominal emergency in 1978 due to ileocolic intussusception. Three separate ileal polyps, each $1.0 \mathrm{~cm}$, were found in the ileum which was resected. She had a stormy postoperative course and died from pulmonary embolus.

Table

\begin{tabular}{lllll}
\hline & Year & Age & Mode of presentation & Site and size of polyps \\
\hline Case 1 & 1967 & 59 & Ileo-ileal intussusception & Ileum, $6.0 \mathrm{~cm}$ \\
& 1969 & 61 & Abdominal pain and vomiting & Gastric antrum, $1.0 \mathrm{~cm} ;$ ileum, $1.5 \mathrm{~cm}$ \\
& 1974 & 66 & Abdominal pain and vomiting & Gastric antrum, $0.5 \mathrm{~cm}$ and $0.5 \mathrm{~cm} ;$ ileum, $0.5 \mathrm{~cm}$ \\
Case 2 & 1978 & 70 & Ileocolic intussusception & Ileum, $1.0 \mathrm{~cm}, 1 \cdot 0 \mathrm{~cm}$, and $1.0 \mathrm{~cm}$ \\
& 1964 & 35 & Ileocolic intussusception & Ileum, $6.0 \mathrm{~cm}$ \\
& 1967 & 38 & Ileo-ileal intussusception & Ileum, $5.0 \mathrm{~cm}$ \\
& 1969 & 40 & Ileocolic intussusception & Ileum, $10.0 \mathrm{~cm}$ amd $1.0 \mathrm{~cm}$ \\
Case 3 & 1982 & 53 & Ileo-ileal intussusception & Ileum, $8.0 \mathrm{~cm}, 1.8 \mathrm{~cm}$, and $1.2 \mathrm{~cm}$ \\
& 1975 & 22 & Ileocaecal intussusception & Ileum, $8.0 \mathrm{~cm}$ and $2.5 \mathrm{~cm}$ \\
& 1981 & 28 & Ileocolic intussusception & Ileum, $5.5 \mathrm{~cm}, 3.5 \mathrm{~cm}, 3.0 \mathrm{~cm}$, and $2.3 \mathrm{~cm}$ \\
\hline
\end{tabular}


CASE 2

Mrs PU, daughter of case 1, first presented in 1964 when she was 35 years of age as an abdominal emergency due to ileocolic intussusception which necessitated a right hemicolectomy. A $6.0 \mathrm{~cm}$ polyp was present in the head of the intussusceptum formed by the terminal ileum.

In 1967 she had a second, ileo-ileal, intussusception due to a $5.0 \mathrm{~cm}$ polyp which was resected.

Two years later, in 1969, she had a third intussusception, this time ileocolic again, which was led by a $10.0 \mathrm{~cm}$ polyp; a small, $1.0 \mathrm{~cm}$ polyp, was also present in the resected length of ileum.

She remained symptom free until 1982 when she once again presented with acute intestinal obstruction. An ileo-ileal intussusception was resected containing three polyps, $8 \cdot 0,1.8$ and $1.2 \mathrm{~cm}$ respectively.

\section{CASE 3}

Mrs LN, daughter of case 2 and grand-daughter of case 1, emigrated to New Zealand in 1972. She first presented in 1975 at the age of 22 with three days of abdominal pain, vomiting, and diarrhoea. At laparotomy an early ileocaecal intussusception was found caused by an $8.0 \mathrm{~cm}$ polyp $5.0 \mathrm{~cm}$ from the ileocaecal valve and a right hemicolectomy was carried out. A smaller, $2.5 \mathrm{~cm}$ polyp was also found close to the ileocaecal valve.

She remained asymptomatic until 1981 when she started to experience episodes of abdominal pain associated with nausea and vomiting. Two weeks after the onset of symptoms she underwent an emergency laparotomy for an ileocolic intussusception, the cause of which was found to be a $5.5 \mathrm{~cm}$ ileal polyp. Three further polyps, $3 \cdot 5,3 \cdot 0$, and $2 \cdot 3$ $\mathrm{cm}$, were identified in the terminal ileum. All were removed with the resected length of gut.

This patient has recently undergone a full gastrointestinal endoscopy with a clear view obtained and no lesions seen.

None of these three patients have been known to have any dietary fads, allergies, or blood eosinophilia. Their serum proteins and immunoglobulin levels have been normal. None of the three have any history of any other illness and, in particular, shown no evidence of gastrointestinal disease or of a connective tissue, skin or bone disorder, or of vasculitis at any time in their lives. Age at first presentation is decreasing, falling roughly by half between generations. Cases 2 and 3 are still alive and well and without symptoms up to two years after the last surgical episode. Detailed chromosome studies were carried out during the last year on cases 2 and 3 and no abnormality was found.

\section{FAMILY STUDIES}

The family tree is shown in Figure 1. It represents a large Devon kindred mainly engaged in occupations connected with agriculture and small town life. They all live in or around the small market town of Tiverton with the exception of case 3 who had emigrated to New Zealand at the age of 19, three years before she developed any symptoms. She is a housewife, married to a printer, and they have four children aged 5 to 12 years who are all well. The disease has affected one female line of the family and numerous siblings from young to old are free from any symptoms. Most of them have been seen and questioned, some repeatedly, in previous years during episodes of hospital admissions of a stricken relative and all were interviewed again during 1982. Few of them have had any significant illness. A daughter of case 2 and a daughter of case 3 have mild asthma. Another daughter of case 2 had cerebral venous thrombosis during pregnancy in 1981; her only child, a boy, died at 4 months with the diagnosis of 'cot death'. On careful questioning no dietary fads or peculiarities of life style could be identified and none of the family ever suffered from any form of gastrointestinal illness. They are aware of recurrent abdominal problems in the family but they do not appear to be unduly anxious about this and we do not feel that it is justified to submit them to radiological or endoscopic investigation in the absence of any symptoms.

\section{PATHOLOGY}

All 22 polyps were submucosal, varying in size from 0.5 to $10.0 \mathrm{~cm}$, the smaller ones being sessile and the larger pedunculated. Three occurred in the gastric antrum (all in case 1) and all these were less than 1.0 $\mathrm{cm}$. Nineteen were in the ileum: only five of these were less than $1.0 \mathrm{~cm}$. All eight intussusceptions involved the ileum, three being ileo-ileal, one ileocaecal, and four ileocolic. All but one of the eight were caused by a polyp $5.0 \mathrm{~cm}$ or larger; the one exception was a close crop of three polyps, each $1.0 \mathrm{~cm}$. The typical macroscopic appearances are illustrated in Figure 2.

The polyps, whether small or large, were histologically identical with little variation in detail. The microanatomy of two small polyps is illustrated in Figure 3 which shows that, though essentially submucosal, these polyps involve the muscularis propria and even the subserosa at an early stage. As they grow larger, they tend to rise above the surface. Ulceration was present in lesions over $2.0 \mathrm{~cm}$. Histologically, all lesions were loose, vascular connective tissue masses the predominant cell being a mononuclear, spindle or stellate, mesenchymal one (Fig. 4). Few were recognisable as mature 


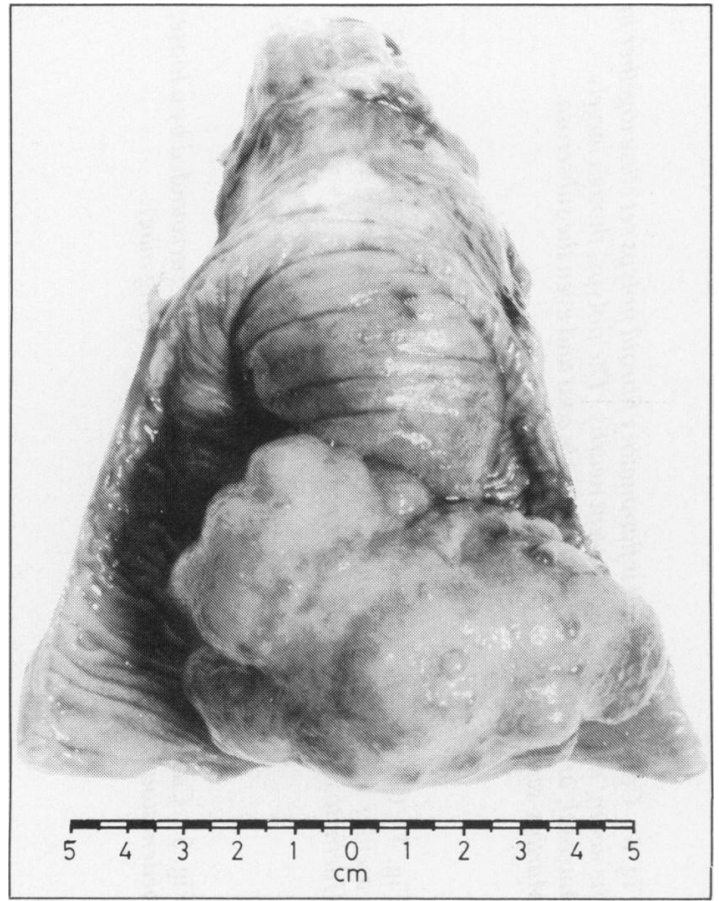

Fig. 2 Case 2. Ileocolic intussusception led by a large inflammatory fibroid polyp in the terminal ileum.

fibroblasts and collagen was scanty. A rich network of reticulum was present, particularly around vessels. These varied from capillary size to thick walled or irregular channels, and were often surrounded by an oedematous mantle (Fig. 5). Eosinophilia was variable and rarely striking; plasma cells, lymphocytes, and mast cells were few. These appearances were identical in every respect with those of 89 solitary cases reviewed by Johnstone and Morson. ${ }^{8}$ None of the lesions were diffuse and in no instances were vasculitis, necrosis, or epitheloid cell granuloma formation seen - that is, none of the features of eosinophilic gastroenteritis $^{10}$ nor of other conditions likely to be confused with inflammatory fibroid polyp. ${ }^{13}$

Electron microscopy was carried out on formalin fixed material from two polyps, both from case 2 (Figs 6 and 7). The appearances were the same as those recorded by Williams ${ }^{14}$ in a single polyp. The predominant cell appeared to be a rather primitive, mesenchymal cell with few cytoplasmic organelles. Mature fibroblasts were few. Capillaries were abundant, in all stages of formation. The intercellular background was loose, with a variable amount of collagen.
Immunohistological studies were carried out on two polyps from each of the three cases. They showed the presence, as expected, of polyclonal IgA and lysozyme in the mucosa surrounding the lesions. The polyps themselves contained few cells that stained positively with any class of immunoglobulin with the exception of IgE in the case of eosinophils. Few mononuclear cells stained positively for $\alpha_{1^{-}}$ antitrypsin, a histiocyte marker, but such cells were strongly positive when antibody to lysozyme, another histiocyte marker, was applied (Fig. 8). Lysozyme was also found, as expected, in neutrophil and eosinophil leucocytes. These results showed no evidence of an abnormality of local immunoglobulin production and they support the predominantly histiocytic nature of the lesion.

\section{Discussion}

An analysis of the clinical presentation of 22 inflammatory fibroid polyps in these three patients reads like reviews of previously published single cases. ${ }^{89}$ There were 10 surgical operations. Eight of these were carried out for the relief of intestinal obstruction owing to intussusception by an ileal lesion; in all but one the polyp was $5.0 \mathrm{~cm}$ or larger. In two instances surgery was made necessary by upper abdominal discomfort and vomiting over several months; in both of these small $(0.5$ to 1.0 $\mathrm{cm}$ ) polyps were present in the gastric antrum. None of the 10 episodes was accompanied by laboratory evidence of an allergy. Johnstone and Morson ${ }^{8}$ recorded blood eosinophilia in only four of 50 cases where this had been looked for and immunoglobulins were normal. These findings are in contrast with those of eosinophilic gastroenteritis and gastrointestinal involvement in collagen vascular diseases with which inflammatory fibroid polyps have been confused in the past. ${ }^{1013}$

The sole identifying feature of inflammatory fibroid polyps is their characteristic histopathology: this was the same in all our cases as that previously described. ${ }^{5-911}$ The main debate on aetiology arises from the microscopic appearances observed and concerns the question whether the lesion is neoplastic or inflammatory. Most authors have considered it an abnormal response to some unknown agent or injury. These polyps are certainly tumour like in being well defined and progressively enlarging up to, in some instances, $10.0 \mathrm{~cm}$ or so. Evidence of inflammation is scanty and consists mainly of a variable infiltrate of eosinophils; the predominant cell type, however, is a mononuclear, mesenchymal one the nature of which is uncertain. Williams $^{14}$ in a careful ultrastructural study of a single polyp found few mature fibroblasts or 


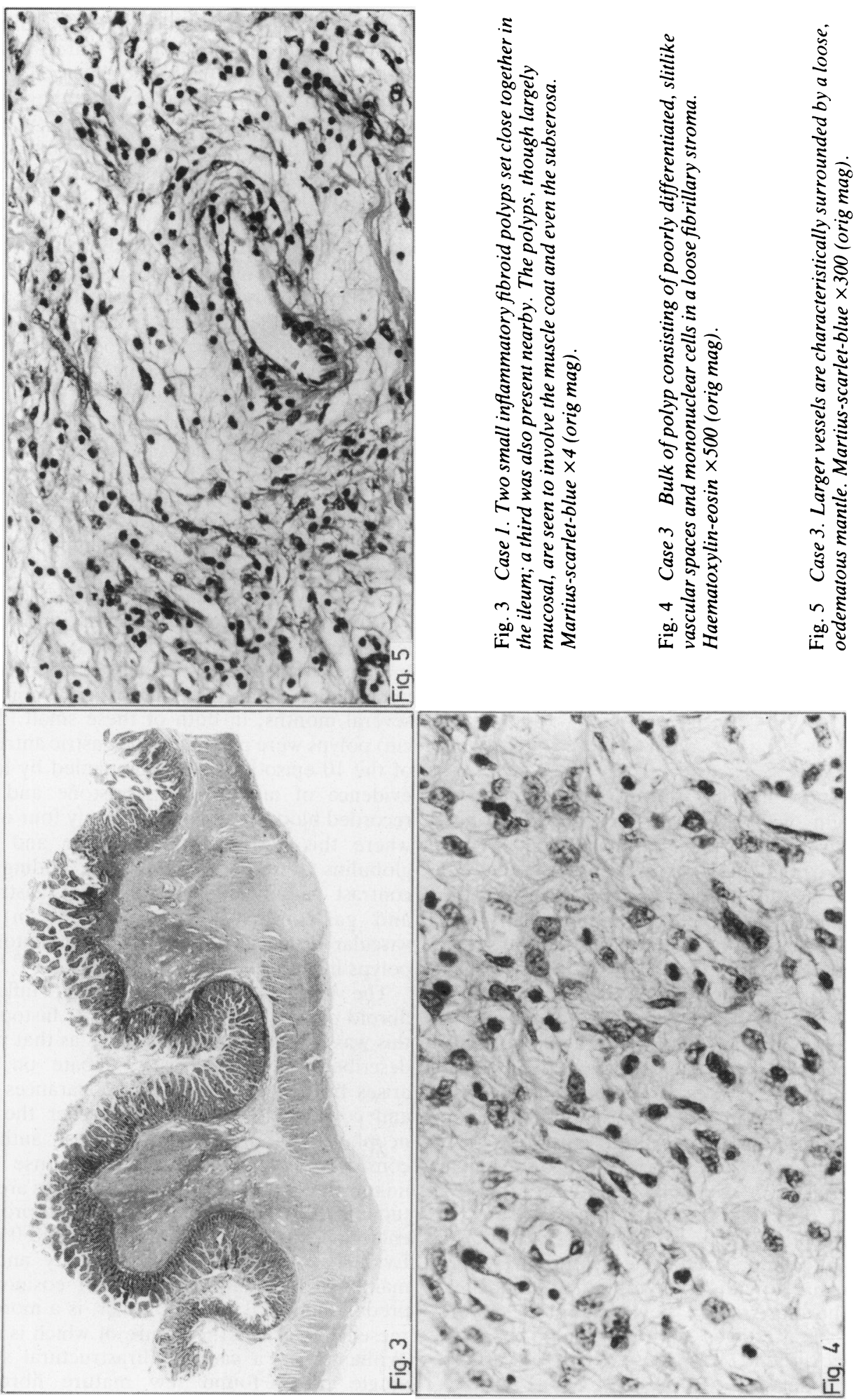




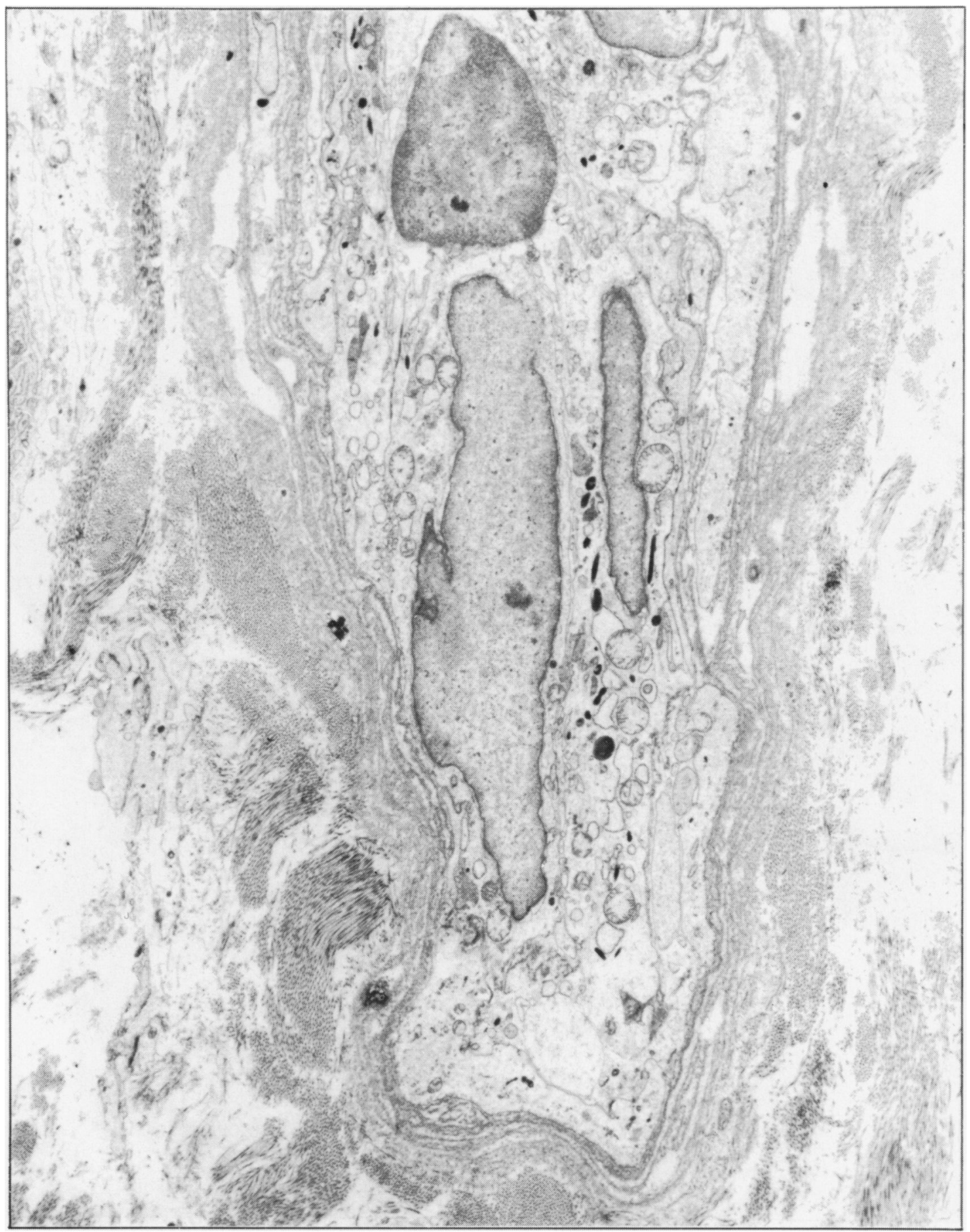

Fig. 6 Case 2. Poorly formed, primitive capillary vessel surrounded a basement membrane and bundles of collagen. EM $\times 7500$ (orig mag). 


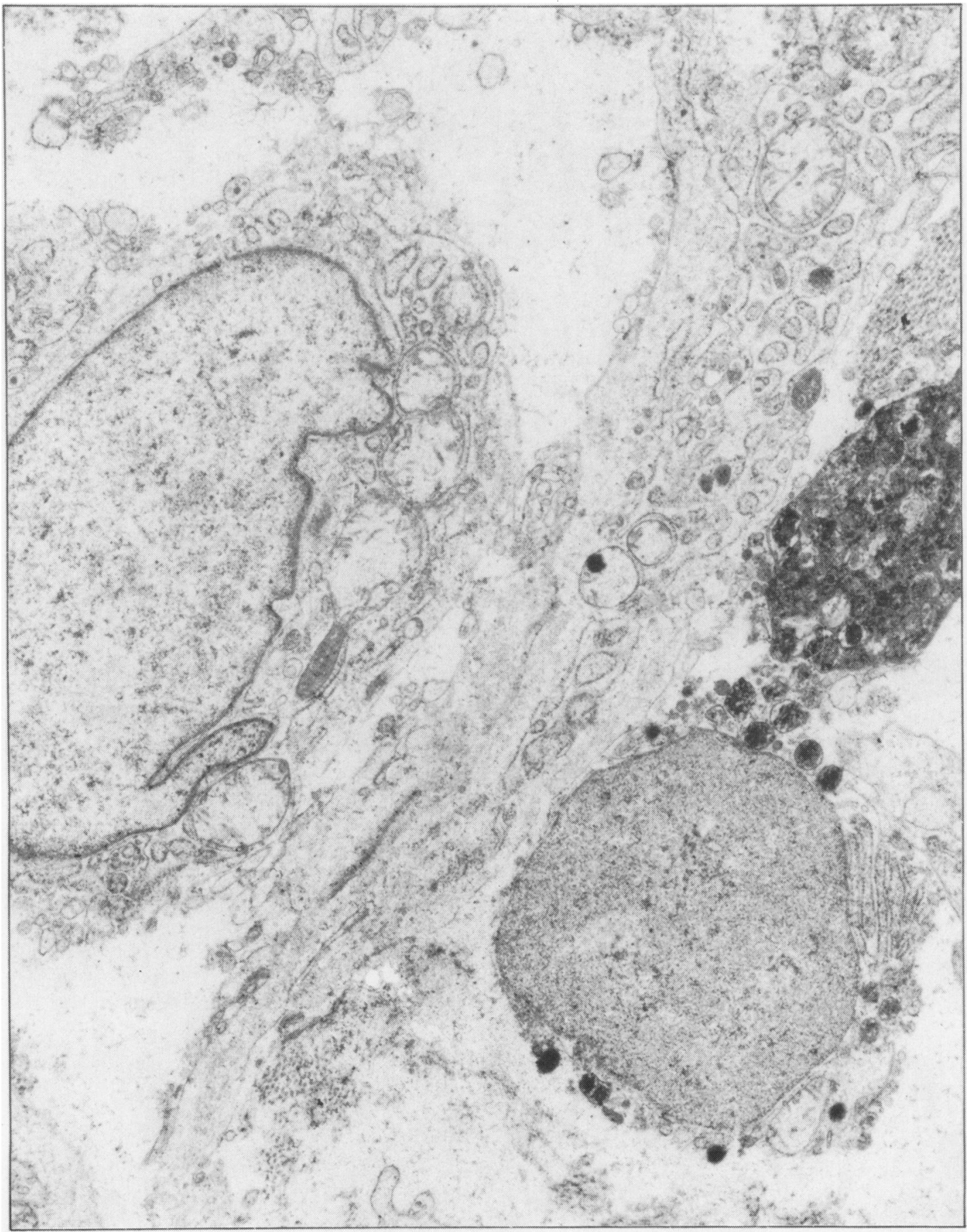

Fig. 7 Case 2 Primitive mesenchymal cells with oddly shaped mitochondria due largely to suboptimal fixation, sparse endoplasmic reticulum, and numerous phagolysosomes (dark). EM $\times 26000$ (orig mag). 


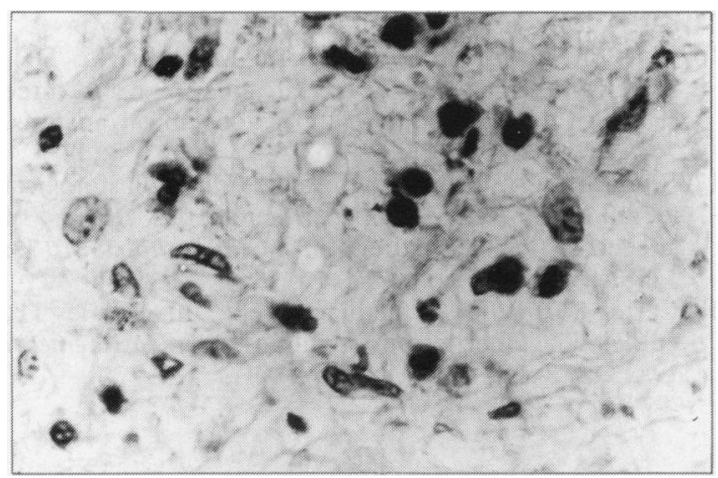

Fig. 8 Case 1. Many mononuclear cells are strongly positive (dark) for lysozyme. Immunoperoxidase $\times 800$ (orig mag).

phagocytic macrophages and emphasised the widespread primitive vascular - that is, endothelial component. We have been able to confirm this on electron microscopy of two polyps. Using immunocytochemistry for the first time for this type of lesion we found, however, that most cells in it carry histiocytic markers, particularly lysozyme which was present in all six polyps examined while $\alpha_{1^{-}}$ antitripsin reactivity was weak or absent. Histiocytic markers are found in premalignant and malignant lymphoproliferative conditions of the gastrointestinal tract and skin. ${ }^{15-17}$ Initially, this is mainly lysozyme and $\alpha_{1}$-antitrypsin is found in the later, malignant phase. These distinctions are not held to be absolute but the suggestion is that inflammatory fibroid polyps are localised, tumour like but self limiting proliferations of histiocytic nature.

The main interest of this report lies in the repeated and multiple occurrence of this rare lesion in three generations of a family: none of these features have been recorded before; indeed, there is only one case record ever of two polyps in the same individual. ${ }^{11}$ There are only two causes of a concentration within families of a disease: common environment and inheritance. ${ }^{18}$ We have been unable to detect an environmental factor that might have been common to members of three successive generations who only lived together in a mother to daughter relationship during childhood and adolescence. The period of time during which all these polyps presented spans 16 years. Case 3 emigrated to New Zealand at the age of 19 and developed the disease in her new environment. Interviews with all living members of the family failed to uncover any dietary fads or peculiarities of life style that might have been handed down. We are left, therefore, with inheritance as the likely mechanism. This could, theoretically, be one of three kinds. A chromosomal anomaly has been excluded in two of the three patients. Such anomalies in any case tend to give rise to multiple, rather than single abnormalities; we have not found any associated pathology. Regular monogenic diseases are recognisable by mendelian patterns indicating dominant, recessive, or $\mathrm{X}$-linked inheritance. Apart from the fact that all three cases occurred in women there is no indication of a Mendelian pattern and numerous siblings remain clinically unaffected. A polygenic type of inheritance seems therefore the most likely and the aetiology may be multifactorial rather than single. The family is clearly unique and their surveillance, particularly of the fourth generation, continues.

We wish to record our indebtedness to Dr Mary Vowles who conducted interviews and drew up the family tree, to Professor A M O Veale and Dr H J R Bussey for advice, and to Professor J F Arthur for making available histological material from case 3 . We thank also Miss Verena White and Mrs M Wood for secretarial assistance.

\section{References}

1 Bussey HJR. Gastrointestinal polyposis syndromes. In: Anthony PP, MacSween RNM, eds. Recent advances in histopathology. Edinburgh: Churchill Livingstone. (In press.)

2 Candy DCA, Hoard FM, McNeish AS. Miscellaneous disorders. In: Harries JT, ed. Familial inherited abnormalities. Clin Gastroenterol 1982; 11: 207-29.

3 Erbe RW. Inherited gastrointestinal-polyposis syndromes. N Engl J Med 1976; 294: 1101-4.

4 Bussey HJR, Veale AMO, Morson BC. Genetics of gastrointestinal polyposis. Gastroenterology 1978; 74: 1325-30.

5 Vanek J. Gastric submucosal granuloma with eosinophilic infiltration. Am J Pathol 1949; 25: 397-411.

6 Helwig EB, Ranier B. Inflammatory fibroid polyps of the stomach. Ann J Pathol 1952; 28: 535-6.

7 Helwig EB, Ranier A. Inflammatory fibroid polyps of the stomach. Surg Gynecol Obstet 1953; 96: 355-67.

8 Johnstone JM, Morson BC. Inflammatory fibroid polyp of the gastrointestinal tract. Histopathology 1978; 2: $349-61$.

9 Namba $T$, Watanabe $H$, Enjoji $M$. Inflammatory fibroid polyp of the stomach. A histological study of 40 cases. Fukuoka Igaku Zasschi 1979; 70: 721-31.

10 Johnstone JM, Morson BC. Eosinophilic gastroenteritis. Histopathology 1978; 2: 335-48.

11 Balmer F, Stucki P, Pedrinis E, Halter F. Tumoren und eosinophile Granulome des Dünndarms bei Malabsorption. Schweiz Med Wochenschr 1974; 104: 78-83. 
12 Burns J. Immunohistological methods and their application in the routine laboratory. In: Anthony PP, Woolf $\mathrm{N}$, eds. Recent advances in histopathology. Edinburgh: Churchill Livingstone, 1978; 10: 337-50.

13 Suen KC, Burton JD. The spectrum of eosinophilic infiltration of the gastrointestinal tract and its relationship to other disorders of angiitis and granulomatosis. Hum Pathol 1979; 10: 31-43.

14 Williams RM. An ultrastructural study of a jejunal inflammatory fibroid polyp. Histopathology 1981; 5: 193-203.

15 Isaacson P. Malignant histiocytosis of the intestine: the early histological lesion. Gut 1980; 21: 381-6.

16 Isaacson P, Jones DB, Sworn MJ, Wright DH. Malignant histiocytosis of the intestine: report of three cases with immunological and cytochemical analysis. $J$ Clin Pathol 1982; 35: 510-6.

17 Burgdorf WH, Duray P, Rosai J. Immunohistological identification of lysozyme in cutaneous lesions of alleged histiocytic nature. Am J Clin Pathol 1981; 75: 162-7.

18 Carter CO. When is familial genetic? In: Harries JT, ed. Familial inherited abnormalities. Clin Gastroenterol 1982; 11: 3-15. 